\title{
Eccentric Macular Hole after Pars Plana Vitrectomy for Idiopathic Macular Hole: A Case Report
}

\author{
Irini Chatziralli George Theodossiadis Maria Douvali \\ Alexandros A. Rouvas Panagiotis Theodossiadis \\ 2nd Department of Ophthalmology, Attikon Hospital, National and Kapodistrian \\ University of Athens, Athens, Greece
}

\section{Keywords}

Eccentric macular hole $\cdot$ OCT · Vitrectomy

\begin{abstract}
Introduction: Postoperative eccentric macular hole (MH) formation is an uncommon complication after pars plana vitrectomy (PPV) with internal limiting membrane (ILM) peeling for epiretinal membrane or $\mathrm{MH}$ treatment. Herein, we present a case of eccentric $\mathrm{MH}$ formation after PPV with ILM peeling for MH. Case Description: A 72-year-old female patient underwent 23-gauge PPV with ILM peeling for idiopathic $\mathrm{MH}$ in her right eye. The visual acuity was $6 / 24$ in the right eye. One week postoperatively the retina was attached and the $\mathrm{MH}$ seemed to be closed, while visual acuity was 6/12. One month after PPV, there was a single eccentric retinal hole below the macula, which was detected at the fundoscopy and was confirmed by OCT. The visual acuity was $6 / 9$ and the patient referred no symptoms. No further intervention was attempted, and at the 6-month follow-up the visual acuity and the size of the eccentric $\mathrm{MH}$ remained stable. Conclusions: Eccentric MHs can develop after PPV and are usually managed conservatively by observation.

(C) 2017 The Author(s)

Published by S. Karger AG, Basel
\end{abstract}




\section{Introduction}

Postoperative eccentric macular hole (MH) formation is an uncommon complication after pars plana vitrectomy (PPV) with internal limiting membrane (ILM) peeling for epiretinal membrane (ERM) or MH treatment. Rubinstein et al. [1] were the first to mention iatrogenic eccentric MHs, and since then only few cases have been reported [2-10]. In fact, the incidence of eccentric MHs ranges between 0.6-2.5\% [1, 3, 10]. Postoperative MHs are located centrally, paracentrally, or eccentrically and are usually single, although multiple MHs have been also observed [1-10]. Herein, we present a case of eccentric MH formation after PPV with ILM peeling for $\mathrm{MH}$.

\section{Case Description}

A 72-year-old female patient presented with blurred vision and metamorphopsia in her right eye. Her ophthalmological as well as medical history was clear. She had undergone cataract surgery in both eyes 3 years previously. At presentation, her best-corrected visual acuity (BCVA) was 6/24 in the right eye and 6/7.5 in the left eye. Intraocular pressure was $12 \mathrm{~mm} \mathrm{Hg}$, and anterior segment slit-lamp examination was unremarkable in both eyes. Dilated fundoscopy revealed idiopathic MH in the right eye, which was confirmed by OCT. The patient was counselled on the therapeutic alternatives and was referred for MH surgery.

Two weeks later, 23-gauge PPV with ILM peeling was performed. Core vitrectomy was followed by surgical creation of posterior vitreous detachment, while Brilliant Blue was used to help ILM peeling. Gas tamponade with $20 \%$ sulfur hexafluoride $\left(\mathrm{SF}_{6}\right)$ was used, and postoperatively the patient was postured face down for 3 days.

One week postoperatively the retina was attached and the MH seemed to be closed, while BCVA was 6/12. One month after PPV, there was a single eccentric retinal hole below the macula, which was detected at the fundoscopy and confirmed by OCT (Fig. 1). BCVA was $6 / 9$ and the patient referred no symptoms. The patient was informed about the presence of the eccentric $\mathrm{MH}$, but no additional intervention was attempted. At the 6-month follow-up, no change in the size of the eccentric $\mathrm{MH}$ was noted and complications occurred.

\section{Discussion}

This is the case of an eccentric MH, which developed about 1 month after PPV with ILM peeling for MH treatment. There is no common consensus about the pathogenesis of postoperative eccentric $\mathrm{MH}$, but various theories have been proposed. The most plausible mechanism could be the iatrogenic trauma, which may occur during surgery when grasping the ILM with forceps [1]. ILM peeling may induce surgical trauma of the underlying nerve fiber layer and Müller cells, causing secondary delayed degeneration of the adjacent retinal neurons [1, 3, 9]. Ultrastructural studies have shown remnants of Müller cell processes attached to the removed ILM with signs of necrosis [3]. However, in the majority of published cases there was no retinal whitening or retinal hemorrhages consistent with postoperative trauma $[1,3,10]$.

A toxic effect of the dyes used for ILM staining may also be considered. Indocyanine green toxicity has been reported after ILM peeling for ERM or MH surgery, but it concerned the retinal pigment epithelium degeneration and was not associated with $\mathrm{MH}$ formation [1, 
10]. In addition, Steven et al. [9] described 7 cases of postoperative paracentral retinal holes after ILM removal in which the retinal holes occurred irrespective of whether indocyanine green, trypan blue, or triamcinolone was used.

Another potential mechanism for the formation of eccentric MHs is the ILM peeling itself. Specifically, ILM removal may result in glial apoptosis and reduced retinal function due to the removal of Müller cells [1]. This may lead to glial structure weakening and consequently to retinal hole development [1, 9]. However, Sandali et al. [3] reported the development of paracentral retinal hole in a patient who did not undergo ILM peeling, although this patient had surgery for epiretinal membrane, and retinal damage could arise from the use of the microvitreous retinal blade in the initial surgical technique, or the firm perifoveal adherence of the hyaloid membrane with resulting retinal traction during ERM removal could be an explanation. Finally, the contraction of residual ILM or ERM could also be a possible cause of eccentric MH development, since MHs are usually located at the limits of the area of ILM peeling [5].

Regarding the treatment of eccentric MHs, there are 3 factors to consider in deciding for further intervention: the visual acuity, the location of the $\mathrm{MH}$, and its evolution in size. Specifically, if the postoperative MH is located near the fovea or is enlarged over time, and if the visual acuity has decreased, surgical intervention may be recommended with gas tamponade and probable additional ILM peeling to allow the MH closure [1, 3]. In our case, no further intervention was needed, and the visual acuity as well as the MH size remained stable at the 6-month follow-up.

\section{Statement of Ethics}

Written informed consent was obtained from the patient to present this case.

\section{Disclosure Statement}

There was no funding for this work. The authors declare no financial disclosure and no conflict of interest.

\section{References}

1 Rubinstein A, Bates R, Benjamin L, Shaikh A: Iatrogenic eccentric full thickness macular holes following vitrectomy with ILM peeling for idiopathic macular holes. Eye 2005;19:1333-1335.

-2 Rush RB, Simunovic MP, Aragon AV 2nd, Ysasaga JE: Postoperative macular hole formation after vitrectomy with internal limiting membrane peeling for the treatment of epiretinal membrane. Retina 2014;34:890-896.

3 Sandali O, El Sanharawi M, Basli E, Lecuen N, Bonnel S, Borderie V, Laroche L, Monin C: Paracentral retinal holes occurring after macular surgery: incidence, clinical features, and evolution. Graefes Arch Clin Exp Ophthalmol 2012;250:1137-1142.

4 Yeh S, Bourgeois KA, Benz MS: Full-thickness eccentric macular hole following vitrectomy with internal limiting membrane peeling. Ophthalmic Surg Lasers Imaging 2007;38:59-60.

5 Mason JO 3rd, Feist RM, Albert MA Jr: Eccentric macular holes after vitrectomy with peeling of epimacular proliferation. Retina 2007;27:45-48.

-6 Abo El Enin MA, El-Toukhy HM, Swelam A: Non-foveal macular holes after PPV for macular pucker.Middle East Afr J Ophthalmol 2010;17:254-256.

7 Sangtam T, Maheshwar B, Au Eong KG: Six cases of eccentric macular holes after vitrectomy with peeling of epimacular proliferation. Retina 2007;27:1321-1322; author reply 1322-1323. 


\section{Case Reports in Ophthalmology}

8 Polkinghorne PJ, Roufail E: Eccentric macular hole formation associated with macular hole surgery. Eye 2007;21:122-123.

-9 Steven P, Laqua H, Wong D, Hoerauf H: Secondary paracentral retinal holes following internal limiting membrane removal. Br J Ophthalmol 2006;90:293-295.

10 Brouzas D, Dettoraki M, Lavaris A, Kourvetaris D, Nomikarios N, Moschos MM: Postoperative eccentric macular holes after vitrectomy and internal limiting membrane peeling. Int Ophthalmol 2016, Epub ahead of print.
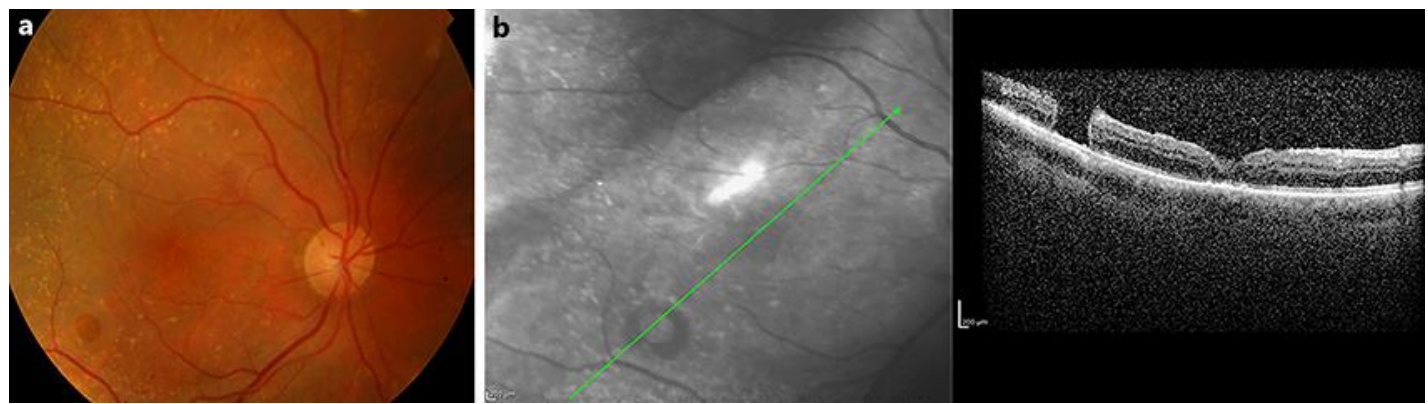

Fig. 1. a Colour fundus photograph. b OCT showing the presence of eccentric macular hole in the right eye of a 72-year-old female patient. 